\title{
Dopamine Operates as a Subsecond Modulator of Food Seeking
}

\author{
Mitchell F. Roitman, ${ }^{1}$ Garret D. Stuber, ${ }^{2}$ Paul E. M. Phillips, ${ }^{1,3}$ R. Mark Wightman, ${ }^{2,3}$ and Regina M. Carelli ${ }^{1,2}$ \\ ${ }^{1}$ Department of Psychology, ${ }^{2}$ Curriculum in Neurobiology, ${ }^{3}$ Department of Chemistry and Neuroscience Center, University of North Carolina at Chapel Hill, \\ Chapel Hill, North Carolina 27599
}

\begin{abstract}
The dopamine projection to the nucleus accumbens has been implicated in behaviors directed toward the acquisition and consumption of natural rewards. The neurochemical studies that established this link made time-averaged measurements over minutes, and so the precise temporal relationship between dopamine changes and these behaviors is not known. To resolve this, we sampled dopamine every $100 \mathrm{msec}$ using fast-scan cyclic voltammetry at carbon-fiber microelectrodes in the nucleus accumbens of rats trained to press a lever for sucrose. Cues that signal the opportunity to respond for sucrose evoked dopamine release ( $67 \pm 20 \mathrm{~nm})$ with short latency $(0.2 \pm 0.1 \mathrm{sec}$ onset). When the same cues were presented to rats naive to the cue-sucrose pairing, similar dopamine signals were not observed. Thus, cue-evoked increases in dopamine in trained rats reflected a learned association between the cues and sucrose availability. Lever presses for sucrose occurred at the peak of the dopamine surges. After lever presses, and while sucrose was delivered and consumed, no further increases in dopamine were detected. Rather, dopamine returned to baseline levels. Together, the results strongly implicate subsecond dopamine signaling in the nucleus accumbens as a real-time modulator of food-seeking behavior.
\end{abstract}

Key words: accumbens; dopamine; feeding; motivation; reward; taste

\section{Introduction}

Foraging for food reward is critical for survival, and brain systems have evolved to direct this behavior. Animals become more efficient at foraging when they learn to associate previously neutral environmental stimuli with food availability. These cues promote motor behavior to obtain food reward (Wyvell and Berridge, 2000). Memory of cue-reward associations, their context, and the animal's emotional state factor into the selection of an appropriate motor response and are encoded in regions such as the hippocampus, prefrontal cortex, and amygdala. These limbic nuclei provide convergent glutamatergic projections to the nucleus accumbens. Indeed, changes in the firing rate of nucleus accumbens neurons encode information related to the operant response for food reward (Carelli, 2002). The nucleus accumbens accesses motor systems that can generate goal-directed behavior (Mogenson et al., 1980), including those involved in feeding (Stratford and Kelley, 1999), and its activity is necessary for feeding-related learning (Smith-Roe and Kelley, 2000).

The dopamine projection from the ventral tegmental area to the nucleus accumbens plays an essential role in modulating goal-directed behavior (Wise et al., 1978). Dopamine filters information by modulating the excitability of nucleus accumbens

Received Aug. 15, 2003; revised Nov. 13, 2003; accepted Dec. 8, 2003

This work was supported by National Institute on Drug Abuse Grants DA10090 to R.M.W. and DA014962 to R.M.C. and R.M.W. and a National Institute of Neurological Disorders and Stroke National Research Service Award to M.F.R. We thank Joyce Wondolowski, Susan Brooks, Michael Heien, Collin McKinney, and John Peterson for technical assistance.

Correspondence should be addressed to Regina M. Carelli, Department of Psychology, Davie Hall CB 3270, University of North Carolina, Chapel Hill, NC 27599-3270. E-mail: rcarelli@unc.edu.

DOI:10.1523/JNEUROSCI.3823-03.2004

Copyright $\odot 2004$ Society for Neuroscience $\quad$ 0270-6474/04/241265-07\$15.00/0 neurons (O'Donnell, 2003) and gating their inputs (Floresco et al., 2001; Horvitz, 2002). Electrophysiological studies have demonstrated that dopamine neurons themselves are transiently activated on a millisecond time scale with presentation of food or food-associated stimuli (Nishino et al., 1987; Schultz, 1998; Hyland et al., 2002). These studies cannot directly assess the extent of dopamine release, however, because it undergoes rich dynamic modulation (Yavich and MacDonald, 2000; Montague et al., 2004). Indeed, there are instances during which driving dopamine neuronal activity can fail to elicit dopamine release altogether (Garris et al., 1999). Nonetheless, there is much evidence that increases in dopamine in the nucleus accumbens play a role in behavior directed at food reward. Microdialysis studies reveal time-averaged increases in extracellular dopamine in the nucleus accumbens over minutes during feeding (Hernandez and Hoebel, 1988; Wilson et al., 1995; Bassareo and Di Chiara, 1999), and dopamine antagonism or depletion disrupts behavior directed at food rewards (Salamone et al., 2003).

The objective of the current study was to make measurements of dopamine during operant responding for food reward on a time scale that can resolve the precise relationship between chemical and individual behavioral events. Rats self-administered intra-oral sucrose by pressing a lever. The lever was accessible at variable intervals by its extension into the chamber and signaled by the illumination of a cue light. Extracellular dopamine was measured every $100 \mathrm{msec}$ throughout the session by electrochemical detection using fast-scan cyclic voltammetry at a carbon-fiber electrode implanted in the nucleus accumbens. Together these methodologies allowed us to study the control of dopamine over cue-evoked, food-seeking behavior, with a level 
of precision not available with traditional neurochemical or pharmacological techniques.

\section{Materials and Methods}

Sucrose self-administration. Male Sprague Dawley rats $(n=5)$ were implanted with intra-oral catheters and after recovery were trained to selfadminister $0.3 \mathrm{~m}$ sucrose during $30 \mathrm{~min}$ daily sessions run at the same time of day each day for each rat from 9:00 A.M. to 1:00 P.M. In the beginning of the session, the chamber was dark; then, after a variable $(30-60 \mathrm{sec})$ delay, the lever extended into the chamber and a cue light illuminated above it. Each lever press resulted in sucrose delivery $(200 \mu \mathrm{l}, 6 \mathrm{sec})$ and a $6 \mathrm{sec}$ tone-light stimulus. This stimulus consisted of a change to general lighting of the chamber from the focal cue light and a continuous auditory tone. After 6 sec the chamber was dark and the next trial began. Once stable responding had been achieved in three consecutive sessions (typically after 8-12 training sessions and 15-22 presses per session across all rats), surgery for fast-scan cyclic voltammetry was performed. Approximately 1 week later, rats were allowed to self-administer sucrose until stable behavior was re-established (typically one session). On the experimental day, rats were permitted to make 15 presses in the same behavioral paradigm while dopamine was monitored in the core of the nucleus accumbens with fast-scan cyclic voltammetry with $100 \mathrm{msec}$ resolution.

Sucrose-naive rats. Another group of rats $(n=3)$ were exposed to daily sessions that differed from sucrose self-administration sessions. After a variable delay $(0.5-32 \mathrm{sec})$ after lever extension and cue-light illumination, the lever retracted, and the tone-light stimulus (computertriggered "press") was presented without sucrose infusion. After a training period comparable to that of the sucrose self-administering rats (eight training sessions), sucrose-naive rats were surgically prepared for voltammetry. Approximately 1 week later, they received 1 more day of training, and then, on the experimental day, dopamine was monitored in the core of the nucleus accumbens during a comparable session of 15 trials per rat.

Voltammetric recording. After training, rats were prepared for voltammetric recording as described previously (Robinson et al., 2002). They were anesthetized with ketamine hydrochloride $(100 \mathrm{mg} / \mathrm{kg}$, i.m.) and xylazine hydrochloride $(20 \mathrm{mg} / \mathrm{kg}, \mathrm{i} . \mathrm{m}$.) and placed in a stereotaxic frame. A guide cannula (Bioanalytical Systems, West Lafayette, IL) was positioned above the nucleus accumbens core $[+1.3 \mathrm{~mm}$ anteroposterior $(\mathrm{AP}), 1.3 \mathrm{~mm}$ mediolateral $(\mathrm{ML})$, and extending $2.5 \mathrm{~mm}$ ventral from bregma]. An $\mathrm{Ag} / \mathrm{AgCl}$ reference electrode was placed in the left forebrain, contralateral to the guide cannula, and all items were secured to the skull with machine screws and cranioplastic cement. A detachable micromanipulator containing a carbon-fiber electrode (50-75 $\mu \mathrm{m}$ length, $7 \mu \mathrm{m}$ diameter cylinders, T-650; Amoco, Greenville, SC) was inserted into the guide cannula, and the electrode was lowered into the nucleus accumbens core. A bipolar stimulating electrode was then placed directly above the ventral tegmental area $(-5.2 \mathrm{~mm} \mathrm{AP,} 1.0 \mathrm{~mm} \mathrm{ML}$ from bregma and $7.5 \mathrm{~mm}$ ventral from dural surface). It was lowered at $0.1-0.2 \mathrm{~mm}$ increments until electrically evoked (60 biphasic pulses, 60 $\mathrm{Hz}, 120 \mu \mathrm{A}, 2 \mathrm{msec}$ per phase) dopamine release was detected at the carbon-fiber electrode. The stimulating electrode was then fixed with cranioplastic cement, and the carbon-fiber electrode was removed.

After voltammetric surgery, all rats were allowed to recover to their presurgery body weight. On the day of the experiment, a new carbonfiber electrode was lowered into the nucleus accumbens core. The carbon-fiber and $\mathrm{Ag} / \mathrm{AgCl}$ reference electrodes were connected to a headmounted voltammetric amplifier attached to a commutator (Med-
Associates, St. Albans, VT) at the top of the test chamber. Voltammetric recordings were made every $100 \mathrm{msec}$ by applying a triangular waveform $(-0.6$ to $+1.4 \mathrm{~V}, 400 \mathrm{~V} / \mathrm{sec})$. Data were digitized and stored to a computer using software written in LabVIEW (National Instruments, Austin, TX). Dopamine release within the nucleus accumbens core was electrically evoked ( 24 biphasic pulses, $60 \mathrm{~Hz}, 120 \mu \mathrm{A}, 2 \mathrm{msec}$ per phase) to insure that carbon-fiber electrodes were in close proximity to dopamine release sites. The electrode position was optimized at a location with maximal dopamine release, and the working electrode was locked in place and allowed to equilibrate for an additional 45-60 min to minimize drift. Experiments were begun when a site was found that had a signalto-noise ratio of electrically evoked dopamine release of at least 30. After the session, an electrical stimulation was repeated to ensure that the site could still support dopamine release. The carbon-fiber electrodes were then removed and calibrated in vitro with $1 \mu \mathrm{M}$ dopamine using a flow injection analysis system (Phillips et al., 2003b).

Signal identification and correction. Cyclic voltammetric data were analyzed on stimulation trials before and after each experiment and $\pm 10 \mathrm{sec}$ relative to the important behavioral events (i.e., cue presentation, lever press). A background signal from 10 voltammetric scans before a stimulation or behavioral trial was subtracted from the remainder of the scans to reveal changes in dopamine concentration (rather than absolute values) (Robinson et al., 2002; Phillips et al., 2003a). For analyte identification, current during a voltammetric scan is plotted against applied potential to yield a cyclic voltammogram (the chemical signature of the analyte). The cyclic voltammogram for dopamine under these conditions is distinct from all anticipated interferences except norepinephrine (Heien et al., 2003). Norepinephrine is unlikely to contribute to our signal for several reasons. First, its synthetic enzyme, dopamine $\beta$-hydroxylase, is present at very low levels in the core of the nucleus accumbens (Berridge et al., 1997). Furthermore, tissue content of norepinephrine in the core is low (2\% of dopamine) (Garris et al., 1993), and our electrode has lower detection sensitivity for it (Heien et al., 2003). The cyclic voltammogram for DOPAC also shares similarities with that for dopamine, but it is unlikely to contribute to the rapid signals detected here because its formation is slow (Fowler and Benedetti, 1983).

Dopamine was identified with anatomical, physiological, and chemical criteria. We histologically verified that all recording sites were within the nucleus accumbens core (Fig. 1), an anatomical region in which dopamine is the major electroactive neurotransmitter. After recording 


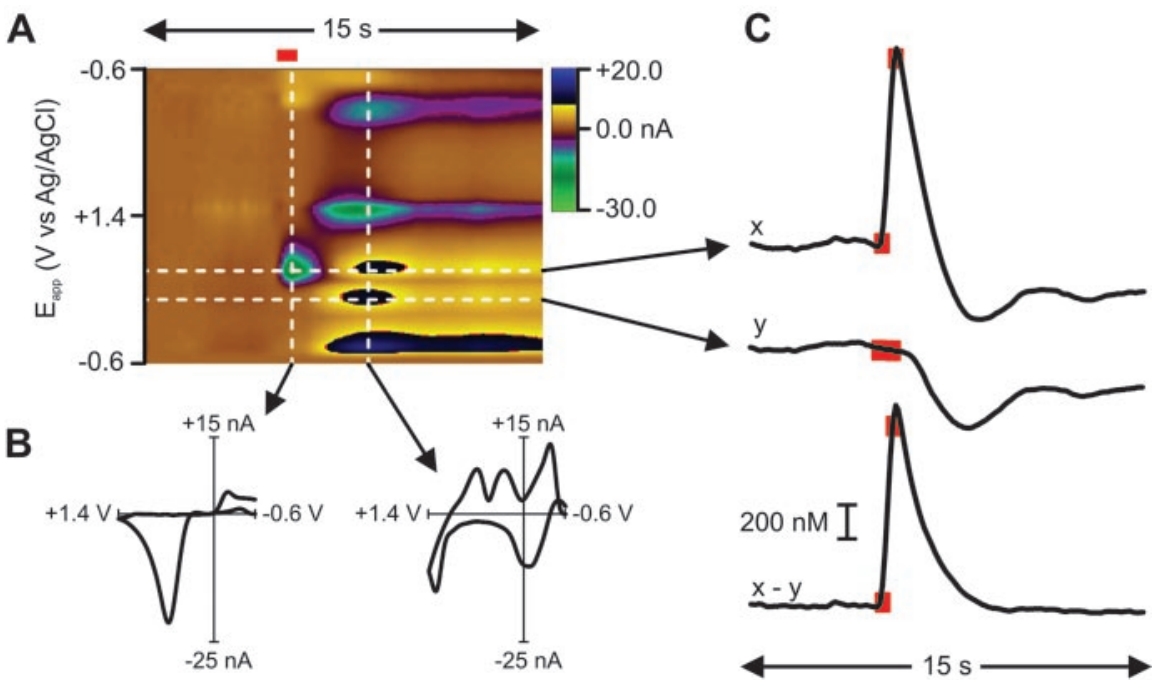

Figure 2. Dopamine release is evoked by stimulation of the ventral tegmental area. The electrochemical data obtained in the nucleus accumbens during a representative electrical stimulation is shown in the color plot $(A)$. The voltammetric current (represented in color) is plotted against the applied potential ( $E_{\text {app }}$; ordinate) for each scan. Consecutive scans (every $100 \mathrm{msec}$ ) are represented along the abscissa (time). Dopamine increased during the stimulation ( 24 biphasic pulses, $60 \mathrm{~Hz}, \pm 120 \mu \mathrm{A}$; red bar). It was identified from its peaks at 0.67 and $-0.20 \mathrm{~V}$. The oxidation peak (green) is accompanied by its reduction peak (yellow). The cyclic voltammogram (current-voltage plot) $(B$, left) extracted from these data are similar to exogenous dopamine in a flow-cell apparatus. The events that take place in the later part of the color plot, and are shown in the right-hand cyclic voltammogram $(B$, right), are clearly not changes in dopamine concentration but more likely a basic pH change in the extracellular space (Venton et al., 2003). The time courses of electrochemical signals taken at different applied potentials are shown in C: $x$ is the current (vs time) at the peak oxidation potential for dopamine (derived from the current-voltage plot), and $y$ is the current at a potential insufficient to oxidize dopamine. Scaling $y$ to the sensitivity at $x$ and subtracting it removes the non-dopaminergic interference from the signal. This is then converted to dopamine concentration by normalization to an in vitro calibration of known dopamine concentration. The red boxes highlight the data points at which electrical stimulation began and ended.

\section{Results}

Electrically evoked dopamine release

Extensive previous characterization has demonstrated that electrical stimulation of the ventral tegmental area causes a transient increase in dopamine in the nucleus accumbens (Garris et al., 1997) and a secondary basic $\mathrm{pH}$ change that correlates with increased local blood flow (Venton et al., 2003). Both of these signals can be detected electrochemically at carbon-fiber microelectrodes and can be separated using fast-scan cyclic voltammetry (Venton et al., 2003). Here, before initiation of behavioral sessions, a carbon-fiber microelectrode was lowered into the core of the nucleus accumbens, and the ventral tegmental area was stimulated (24 biphasic pulses, $60 \mathrm{~Hz}, 120 \mu \mathrm{A}, 2 \mathrm{msec}$ per phase) to confirm that terminals in the microenvironment around the electrode were capable of rapid dopamine release. A representative stimulation trial is shown in Figure 2. Dopamine increased during electrical stimulation of the ventral tegmental area as confirmed by its oxidation and reduction peaks at 0.67 and $-0.20 \mathrm{~V}$, respectively. The cyclic voltammogram (Fig. $2 B$, left current-voltage plot) extracted from these data is similar to exogenous dopamine in a flow-cell apparatus. After the stimulation was terminated, a second chemical change at the recording site took place. The change was clearly not caused sessions, rats were anesthetized deeply, and a stainless steel electrode (50 $\mu \mathrm{m}$ tip radius) housed in the same micromanipulator used during the experiment was lowered to the experimental recording site and a small electrolytic lesion was made ( $50 \mu \mathrm{A}, 10 \mathrm{sec}$ ). Rats were then perfused transcardially, and brains were removed. After postfixing, brains were sliced on a freezing cryostat, and sections were mounted on slides and stained with potassium ferrocyanide (to form a blue reaction product) and counterstained with thionin to reveal the location of the lesion. Stimulation of dopamine cell bodies before and after behavioral sessions provided physiological evidence that the recording site was an area that could support rapid dopamine release. Detection of dopamine, revealed by the cyclic voltammograms, occurred at all recording sites. Chemical evidence for dopamine was provided by the cyclic voltammogram, which offers information specific to the analyte. The cyclic voltammograms observed around the lever press, cues, and sucrose delivery were compared statistically with those from electrical stimulations at the same recording site and those from in vitro calibration of the electrode. For every animal, the responses around cue-light illumination-lever extension and lever press were averaged. The changes in signal were attributed to dopamine if the cyclic voltammogram closely correlated with that for stimulated dopamine release $\left(r^{2}>0.75\right)$.

To reveal the temporal profile of dopamine changes, the corrected dopamine oxidation current was plotted against time. The corrected current was obtained by subtraction of electrochemical current at a potential that included interference (for example, $\mathrm{pH}$ changes or movement artifacts) but at which oxidation of dopamine did not contribute (around $+0.20 \mathrm{~V}$ against $\mathrm{Ag} / \mathrm{AgCl}$ ) (Venton et al., 2003) from the peak dopamine oxidation potential (around $+0.70 \mathrm{~V}$ against $\mathrm{Ag} / \mathrm{AgCl}$; includes dopamine and the interference). Dopamine concentration changes were estimated from this current by calibration of the electrode after in vivo use. by changes in dopamine concentration (Fig. $2 \mathrm{~B}$, right currentvoltage plot) but more likely by a basic $\mathrm{pH}$ change in the extracellular space (Venton et al., 2003). As mentioned in Materials and Methods, this interference was subtracted out of the dopamine trace. Experiments were initiated when robust increases in dopamine were elicited (at least 30:1 signal-to-noise ratio).

\section{Dopamine response to sucrose-predictive cues during self-administration}

To investigate phasic dopamine release during feeding behavior, fast-scan cyclic voltammetry was used in five rats well trained to self-administer sucrose. Before each trial, the chamber was dark and the lever was retracted. After a variable delay $(30-60 \mathrm{sec})$, the lever extended into the chamber, and a cue light was illuminated over it. When the rat pressed the lever, the cue light extinguished and the lever retracted. In addition, the infusion pump turned on for $6 \mathrm{sec}$ to deliver sucrose intra-orally $(200 \mu \mathrm{l})$ paired with a tone-light stimulus. Cue-light illumination-lever extension evoked subsecond increases in extracellular dopamine, an example of which can be seen in Figure 3. Here, after cue-light illumination-lever extension (indicated by arrowhead), there was an immediate rise in signal that peaked at $0.3 \mathrm{sec}$. The change in signal was confirmed to be caused by an increase in extracellular dopamine concentration because dopamine was identified from its oxidation and reduction peaks at 0.66 and $-0.22 \mathrm{~V}$, respectively, in the color plot and in the cyclic voltammogram. A statistical comparison of this cyclic voltammogram with one from electrically evoked dopamine release from the same rat (Fig. 2) 


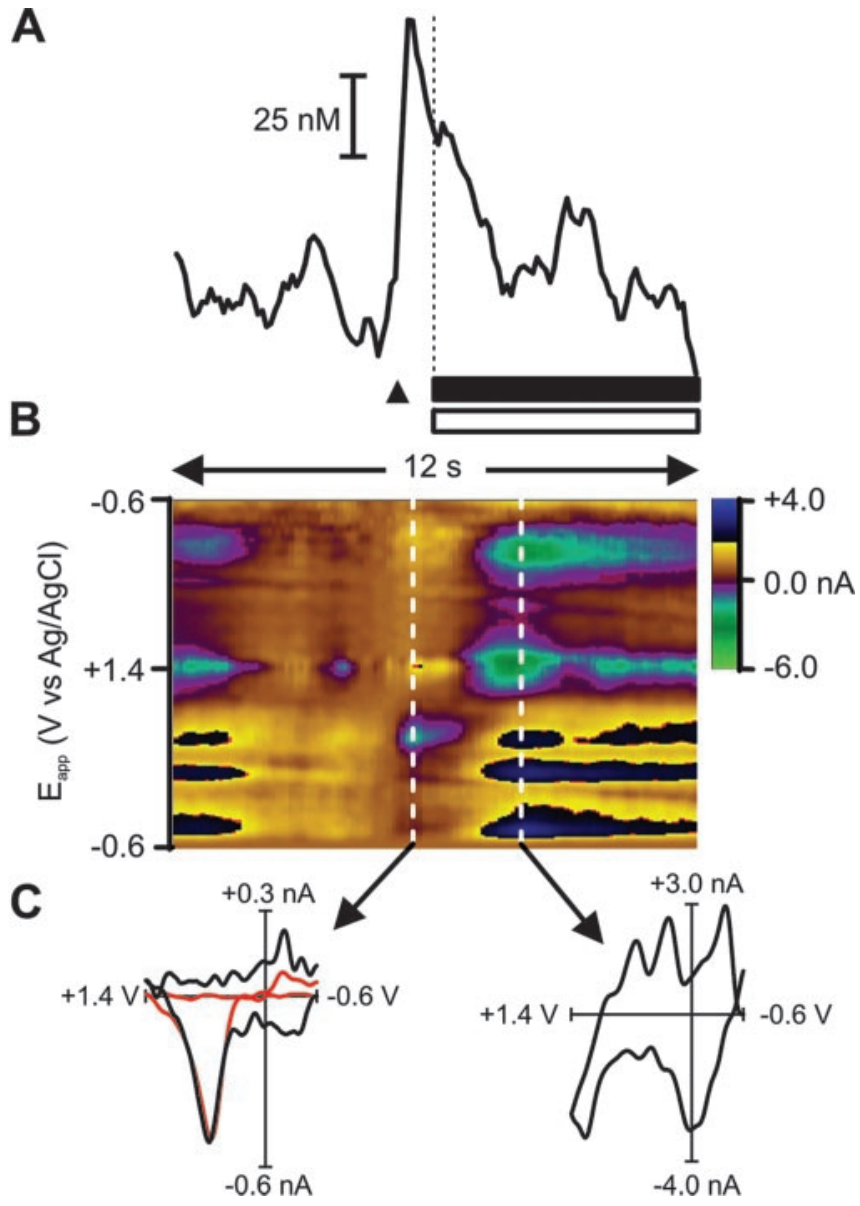

Figure 3. Cue presentation elicits a phasic surge in dopamine. $A$, The rise in signal evoked by a representative cue presentation (denoted by black triangle). The peak in dopamine concentration occurred just before the operant response for intra-oral sucrose (denoted by vertical dashed line) and fell to baseline levels during the duration of the intra-oral infusion (black bar) and tone-light stimulus (open bar). B, Dopamine was identified from its peaks at 0.66 and $-0.22 \mathrm{~V}$ and in the cyclic voltammogram ( $C$, left). As can be seen in the color plot, the oxidation peak (green) is accompanied by its reduction peak (yellow). A statistical comparison of this cyclic voltammogram with one from electrically evoked dopamine release (normalized and shown in red) reveals a close correlation $\left(r^{2}=0.77\right)$. The events that take place in the later part of the color plot, and shown in the right-hand cyclic voltammogram (C, right), are clearly not changes in dopamine concentration. This cyclic voltammogram was not correlated with the one from electrically evoked dopamine release $\left(r^{2}=0.16\right)$. Rather, the events in the latter part of the color plot are more likely caused by a basic $\mathrm{pH}$ change in the extracellular space. This interference was removed from the signal trace in the same manner as described for removing interference from electrically evoked dopamine release (Fig. 2).

performed before the behavioral session (normalized and shown in red in Fig. 3) reveals a close correlation $\left(r^{2}=0.77\right)$.

The average response for the five rats used in this experiment (15 trials per rat), as seen in Figure 4 (top), was that of a rise in dopamine $>2$ SDs above mean baseline (10 sec period before cue-light illumination-lever extension). The dopamine response to cue-light illumination-lever extension occurred with very short latency $(0.2 \pm 0.1 \mathrm{sec}$ onset $)$ and peaked within $1 \mathrm{sec}$ of onset of the cues $(0.7 \pm 0.1 \mathrm{sec})$. To validate the increase in signal as a rapid increase in extracellular dopamine, a cyclic voltammogram, taken at the peak of the signal on each trial for each rat, was averaged for all rats and compared statistically with the averaged cyclic voltammogram for electrically evoked dopamine release. The averaged cyclic voltammogram obtained from the increase in signal after cue-light illumination-lever extension (Fig. 4, top inset) was tightly correlated $\left(r^{2}=0.87\right)$ with the averaged cyclic

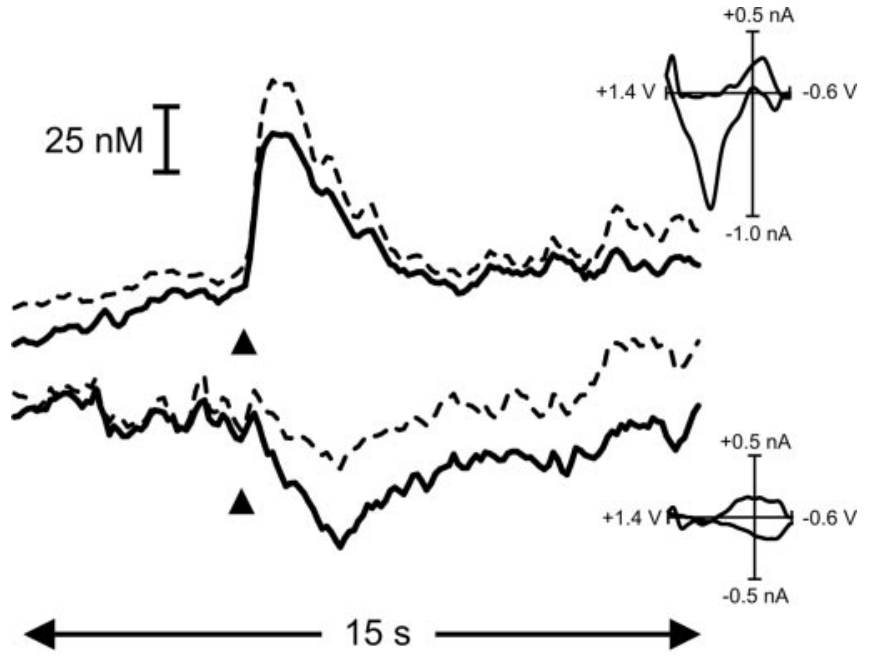

Figure 4. Cue presentation evokes phasic surges in dopamine and reflects a learned association. Increases in signal (mean + SEM represented by solid and dashed black lines, respectively) were evoked by cue presentation (denoted by black triangle) for operant responding rats ( $n=5$; top trace). The robust increase in signal was confirmed to be a rise in dopamine concentration by examination of the averaged cyclic voltammogram taken at the peak of the signal (inset). Cue presentation failed to elicit an increase in dopamine in rats ( $n=3$; bottom trace) that did not have the cue-sucrose pairing. The decrease in signal just after cue presentation was not caused by a change in dopamine. This was confirmed by comparison of the averaged cyclic voltammogram (inset) with the averaged cyclic voltammogram taken from pre-session, electrically evoked dopamine release $\left(r^{2}<0.01\right)$. The signal in operant responding rats was significantly higher than control rats immediately after cue presentation $(0.5-2.4$ sec; $p<0.05$; post hoc Student's $t$ test with Bonferroni correction).

voltammogram taken from electrically evoked dopamine release, establishing dopamine as the detected substance.

\section{Dopamine response to cues in inexperienced rats}

The increase in dopamine after cue-light illumination-lever extension may reflect a learned association between the cues and the availability of sucrose or merely the salient change in the environment independent of reward (Horvitz, 2000). To resolve this, a control group of rats experienced the same number of "training" sessions before dopamine sampling. In these sessions the chamber was dark, and after a variable delay $(30-60 \mathrm{sec})$ the cue light was illuminated and the lever extended into the chamber. After another variable delay $(0.2-16.0 \mathrm{sec})$, the lever was retracted, the tone-light stimulus was played, and the pump was turned on ("computer-triggered press"), but no sucrose was delivered. Thus, the session had two key differences from the operant one: (1) no operant was made, and (2) rats never experienced sucrose. Contrary to the operant-responding rats, in these sessions, dopamine release was not time locked to the cue-light illuminationlever extension [two-way ANOVA significant time by experience interaction $\left(F_{(200,1206)}=2.09 ; p<0.0001\right.$ (Fig. 4)] or the "computer-triggered press" [two-way ANOVA significant time by experience interaction $\left(F_{(200,1206)}=1.31 ; p<0.005\right.$; data not shown)]. This suggests that the dopamine response to the cues that we observed in operant-responding rats is dependent on the learned association with the availability of sucrose.

Relationship between dopamine and the behavioral response For all trials, a lever-press response followed cue-light illumination-lever extension $(5.5 \pm 4.0 \mathrm{sec}$ latency to press; mean \pm SEM). In $83 \%$ of trials ( 62 of 75 ), this occurred within $5 \mathrm{sec}$ of the cue-light illumination-lever extension (average $1.2 \pm 0.1 \mathrm{sec}$ ), 


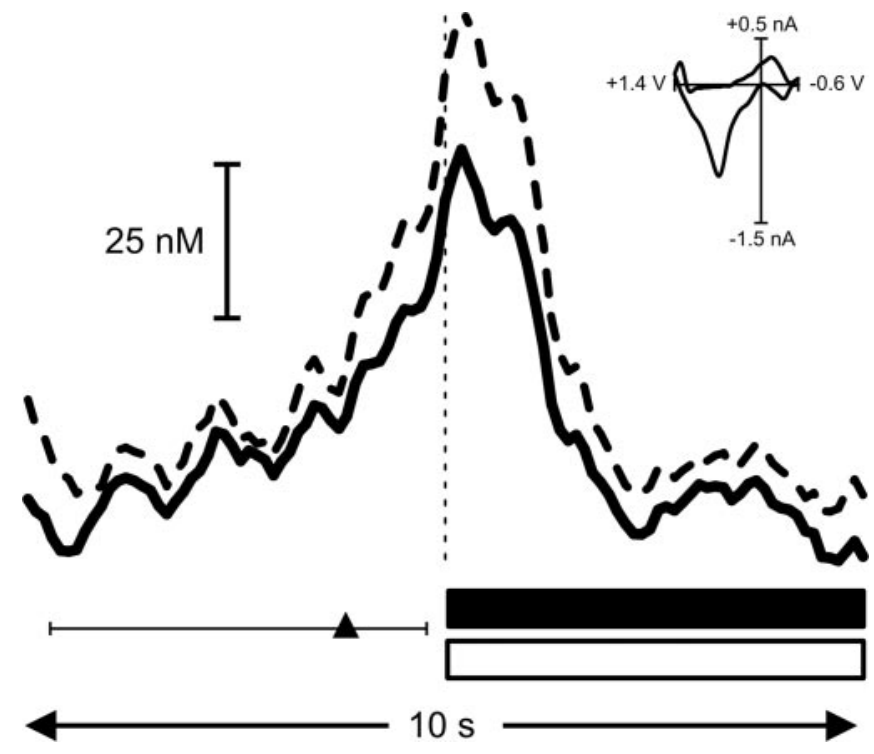

Figure 5. Rats lever press at the peak of cue-evoked dopamine release. Increases in signal (mean + SEM represented by solid and dashed black lines, respectively) began immediately before and peaked at the operant response (denoted by vertical dashed line) on trials when cue presentation elicited an immediate behavioral response ( $<5$ sec latency to press). The average time of cue presentation is denoted by the black triangle, and the range of times is represented by the horizontal scale bar. The increase in signal at the time of the lever press was confirmed to be dopamine by comparison of the averaged cyclic voltammogram taken at the peak of the signal (inset) with the averaged cyclic voltammogram from pre-session electrically evoked dopamine release $\left(r^{2}=0.90\right)$. Dopamine concentration rapidly returned to baseline levels during sucrose infusion (horizontal black bar) and tone-light presentation (horizontal open bar) and remained stable thereafter.

and thus the cue was effective in "immediately" eliciting a goaldirected behavior. This type of rapid behavioral response was evident in all of the rats used here. The average change in extracellular dopamine concentration aligned to the lever press for these "short-latency" trials is shown in Figure 5. The arrowhead depicts the average time at which cue-light illumination-lever extension occurred, and the line through the arrowhead depicts the range of times that these cues were presented relative to the lever press. It should be noted that the signal rises at several points during the time at which cues are presented, reflecting the response shown in Figure 4. Here, when these same trials are aligned to the time at which rats made their operant responses, it becomes evident that responses were made at the height of dopamine increases. On these trials, the lever press occurred close to the peak $(0.7 \pm 0.3 \mathrm{sec})$ of the dopamine response evoked by the cue (Fig. 4). Notably, the peak dopamine concentration was statistically indistinguishable $(68 \pm 19 \mathrm{~nm}$ for cues vs $73 \pm 23 \mathrm{~nm}$ for press) when aligned to either of these behavioral events.

In the remaining $17 \%$ of trials ( 13 of 75 ), the cue failed to elicit an "immediate" behavioral response (average latency to lever press, $26.2 \pm 10.2 \mathrm{sec}$ ). These "long-latency" trials were evident in three of the five rats used in this experiment (one, three, and nine responses contributing from the three rats). On these trials, the dopamine response after cue-light illumination-lever extension was weaker than on short-latency trials. Moreover, there was a second, more robust increase in dopamine that began just before and peaked at the press (Fig. 6). The time course of this second dopamine response with respect to lever pressing was very similar to that observed on short-latency trials. A statistical comparison showed significant differences between peak dopamine concentration observed at cue-light illumination-lever extension (54 \pm

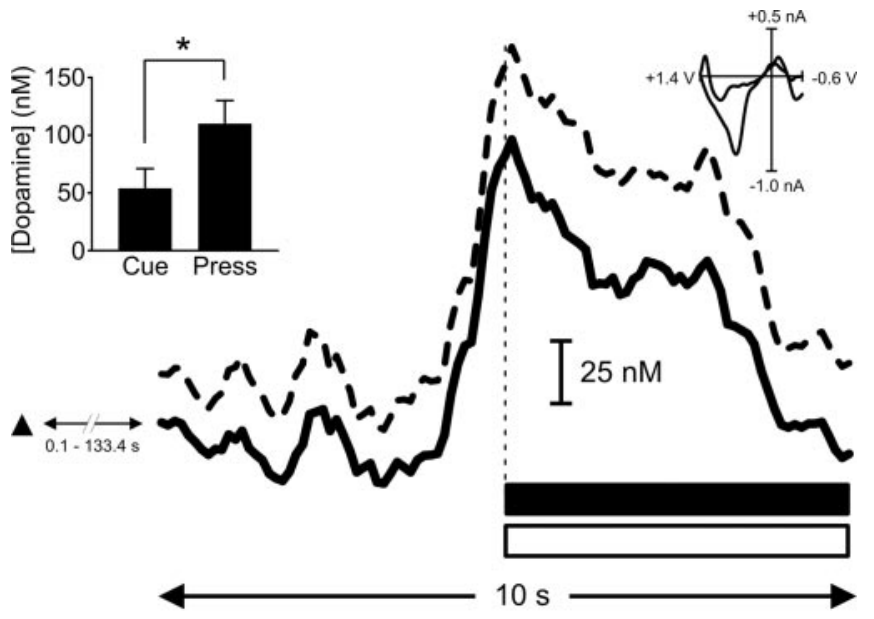

Figure 6. Even when cues fail to elicit an immediate behavioral response, rats lever press at a dopamine peak. A dramatic increase in signal (mean + SEM represented by solid and dashed black lines, respectively) was observed immediately before and peaked at the operant response (denoted by vertical dashed line) on trials when cue presentation failed to elicit an immediate behavioral response ( $>5$ sec latency to press). The cue presentation (black triangle) was at a time $(0.1-133.4 \mathrm{sec})$ before the start of the trace. Cue presentation elicited a rise in dopamine, but rats did not press the lever until a second, larger rise in dopamine occurred $\left({ }^{*} p<0.05\right.$; left inset). The large rise in signal was confirmed to be dopamine release by comparison of averaged cyclic voltammogram (right inset) taken from the peak of the signal with that from pre-session, electrically evoked dopamine release $\left(r^{2}=0.87\right)$. Dopamine concentration returned to baseline levels during sucrose infusion (horizontal black bar) and tone-light presentation (horizontal open bar).

$17 \mathrm{~nm})$ compared with that at the press $(110 \pm 20 \mathrm{~nm} ; p<0.05$; Student's $t$ test) (Fig. 6).

\section{Discussion}

There is widespread agreement that dopamine in the nucleus accumbens participates in some aspects of feeding behavior; however, its specific role in the individual components of the behavior (i.e., approach, response, consumption) remains fervently debated. To address this, we measured dopamine throughout entire sequences of feeding behavior with unprecedented time resolution that matches the endogenous physiology. Presentation of the cues denoting sucrose availability evoked phasic increases in dopamine in the nucleus accumbens. This had a short latency akin to the activation of dopamine neurons on presentation of food-predictive cues (Schultz, 1998) and to dopamine increases for cocaine-predictive cues (Phillips et al., 2003a). Dopamine neurons, however, can respond to salient sensory stimuli that lack conditioned motivational properties (Horvitz, 2000), which could account for the changes that we observed. This possibility was ruled out when the same cues were presented to a control group of animals. These rats had an equal number of training sessions as the operant rats and thus had the same amount of cumulative experience with the cues except that they were never paired to sucrose. Dopamine was evoked by the cues only in rats in which they had been paired to the reinforcer and thus reflected a learned association between the cues and sucrose reward.

There is evidence that rapid increases in dopamine can promote reward seeking (Phillips et al., 2003a). Here, rewardseeking behavior (culminating in a lever press) occurred shortly after the rise in extracellular dopamine, typically close to its peak; however, although it is clear that the dopamine release was evoked by the cue and that the cue ultimately precipitated the behavioral reaction, whether dopamine mediated the cue-evoked 
behavior is less evident from these trials. Further insight can be gained from trials in which the cue failed to rapidly evoke a behavioral response. In these trials, dopamine release was evoked by the cue; however, the operant response was time locked to a second dopamine increase up to 2 min later. This has similarities to our previous report in which electrically evoked dopamine release produced drug seeking only for a subset of stimulation events (Phillips et al., 2003a) and may reflect the fact that dopamine is modulating the circuit rather than driving it directly. Moreover, the remarkable concurrence of a dopamine rise with the initiation of food seeking, even when not immediately preceded by cue-light illumination-lever extension, strongly implicates its role in promoting goal-directed behaviors.

During the delivery of sucrose, the primary reinforcer, dopamine did not increase. Rather, its levels returned to baseline shortly after the response and remained relatively stable thereafter. Dopamine has been thought to mediate the rewarding aspects of food (Wise et al., 1978). More recently, studies have demonstrated a more complicated association between rapid dopamine neurotransmission and food reward. For predicted food rewards in well trained monkeys, dopamine neurons respond to the cues that predict food delivery rather than the food itself (Schultz, 1998). Normal dopamine signaling does not appear to be necessary for food consumption (Salamone et al., 2002) or the orofacial responses that reflect "liking" of food stimuli (Berridge and Robinson, 1998). Our results in well trained rats, together with these findings, demonstrate a clear dissociation between some types of dopamine signaling and the primary aspects of food reward. It now seems to be a general finding that, in experienced animals, dopamine neuronal activity (Schultz et al., 1993; Schultz, 1998) and nucleus accumbens dopamine release are not increased by the presentation and consumption of expected food reinforcers.

In some earlier studies there was an electrochemical change that purports to be a decrease in dopamine in the period after the operant response for food (Kiyatkin and Gratton, 1994; Richardson and Gratton, 1996). Likewise, during some trials in this study, electrochemical changes (typically long-lasting decreases) were observed after the response; however, the additional chemical information obtained with fast-scan cyclic voltammetry over other rapid electrochemical techniques (Phillips and Wightman, 2003) afforded us the insight that these were not changes in dopamine and allowed us to remove them from confirmed dopamine signals. In fact, dopamine levels returned to baseline shortly after the response and remained relatively stable thereafter. Similar non-dopaminergic signals can follow electrical stimulations and have been characterized as basic $\mathrm{pH}$ changes that are tied to changes in neural activity (Venton et al., 2003).

Rapid increases in extracellular dopamine preceded the operant response by hundreds of milliseconds for both sucrose (reported here) and cocaine (Phillips et al., 2003a). For cocaine, rats had access to the lever at all times but approached and responded typically once every $5 \mathrm{~min}$. Here, we were able to study a more defined approach period by limiting access to the lever. It is striking that phasic dopamine is time locked to approach behavior for both cocaine and sucrose because it points to a specific role for dopamine in response initiation regardless of reinforcer. Where these data differ, however, is that dopamine quickly returned to baseline levels after the operant response for sucrose, but there was another rise in dopamine for cocaine. This could reflect an inherent difference between drug and natural reinforcement but may relate to other experimental differences. For instance, the predictive strength of the cues signaling reinforcer delivery may differ. Intravenously delivered cocaine has delayed onset of action (Pan et al., 1991), and so the tone-light cue precedes reinforcement and can predict impending cocaine reward. Intra-oral sucrose, however, results in almost immediate reinforcement (Grill and Norgren, 1978), and so the post-response cues are redundant and have no predictive strength (Waelti et al., 2001).

It has been proposed that dopamine, in the nucleus accumbens, encodes incentive stimuli (Berridge and Robinson, 1998). In well trained animals, dopamine appears to be critical for instrumental actions rather than consumption (Salamone, 1996) and gates responses to favor a high-yield return even when work requirements are higher (Salamone and Correa, 2002). In support of these classic views, we now demonstrate that dopamine explicitly changes at the exact times when these facets of behavior occur. Specifically, we show that dopamine is strongly evoked by incentive stimuli, the cue-light illumination-lever extension, and that this neurochemical change promotes the operant response for sucrose reward. This high extracellular concentration of dopamine at the time of response initiation may serve to gate glutamatergic afferents and therefore select the appropriate motor output for the given sensory input.

\section{References}

Bassareo V, Di Chiara G (1999) Differential responsiveness of dopamine transmission to food-stimuli in nucleus accumbens shell/core compartments. Neuroscience 89:637-641.

Berridge CW, Stratford TL, Foote SL, Kelley AE (1997) Distribution of dopamine beta-hydroxylase-like immunoreactive fibers within the shell subregion of the nucleus accumbens. Synapse 27:230-241.

Berridge KC, Robinson TE (1998) What is the role of dopamine in reward: hedonic impact, reward learning, or incentive salience? Brain Res Rev 28:309-369.

Carelli RM (2002) Nucleus accumbens cell firing during goal-directed behaviors for cocaine vs. "natural" reinforcement. Physiol Behav $76: 379-387$.

Floresco SB, Blaha CD, Yang CR, Phillips AG (2001) Modulation of hippocampal and amygdalar-evoked activity of nucleus accumbens neurons by dopamine: cellular mechanisms of input selection. J Neurosci 21:2851-2860.

Fowler CJ, Benedetti MS (1983) The metabolism of dopamine by both forms of monoamine oxidase in the rat brain and its inhibition by cimoxatone. J Neurochem 40:1534-1541.

Garris PA, Collins LB, Jones SR, Wightman RM (1993) Evoked extracellular dopamine in vivo in the medial prefrontal cortex. J Neurochem 61:637-647.

Garris PA, Christensen JRC, Rebec GV, Wightman RM (1997) Real-time measurement of electrically evoked extracellular dopamine in the striatum of freely moving rats. J Neurochem 68:152-161.

Garris PA, Kilpatrick M, Bunin MA, Michael D, Walker QD, Wightman RM (1999) Dissociation of dopamine release in the nucleus accumbens from intracranial self-stimulation. Nature 398:67-69.

Grill HJ, Norgren R (1978) The taste reactivity test. I. Mimetic responses to gustatory stimuli in neurologically normal rats. Brain Res 143:263-279.

Heien MLAV, Phillips PEM, Stuger GD, Seipel AT, Wightman RM (2003) Overoxidation of carbon-fiber microelectrodes enhances dopamine adsorption and increases sensitivity. Analyst 128:1413-1419.

Hernandez L, Hoebel BG (1988) Food reward and cocaine increase extracellular dopamine in the nucleus accumbens as measured by microdialysis. Life Sci 42:1705-1712.

Horvitz JC (2000) Mesolimbocortical and nigrostriatal dopamine responses to salient non-reward events. Neuroscience 96:651-656.

Horvitz JC (2002) Dopamine gating of glutamatergic sensorimotor and incentive motivational input signals to the striatum. Behav Brain Res 137:65-74.

Hyland BI, Reynolds JNJ, Hay J, Perk CG, Miller R (2002) Firing modes of midbrain dopamine cells in the freely moving rat. Neuroscience 114:475-492.

Kiyatkin EA, Gratton A (1994) Electrochemical monitoring of extracellular 
dopamine in nucleus accumbens of rats lever-pressing for food. Brain Res 652:225-234.

Mogenson GJ, Jones DL, Yim CY (1980) From motivation to action: functional interface between the limbic system and the motor system. Prog Neurobiol 14:69-97.

Montague PR, McClure SM, Baldwin PR, Phillips PEM, Budygin EA, Stuber GD, Kilpatrick MR, Wightman RM (2004) Dynamic gain control of dopamine delivery in freely moving animals. J Neurosci, in press.

Nishino H, Ono T, Muramoto K, Fukuda M, Sasaki K (1987) Neuronal activity in the ventral tegmental area (VTA) during motivated bar press feeding in the monkey. Brain Res 413:302-313.

O’Donnell P (2003) Dopamine gating of forebrain neural ensembles. Eur J Neurosci 17:429-435.

Pan HT, Menacherry S, Justice JB Jr (1991) Differences in the pharmacokinetics of cocaine in naive and cocaine-experienced rats. J Neurochem 56:1299-1306.

Paxinos G, Watson C (1998) The rat brain in stereotaxic coordinates. Orlando, FL: Academic.

Phillips PEM, Wightman RM (2003) Critical guidelines for validation of the selectivity of in-vivo chemical microsensors. Trends Anal Chem 22:509-514.

Phillips PEM, Stuber GD, Heien MLAV, Wightman RM, Carelli RM (2003a) Subsecond dopamine release promotes cocaine seeking. Nature 422:614-618.

Phillips PEM, Robinson DL, Stuber GD, Carelli RM, Wightman RM (2003b) Real-time measurements of phasic changes in extracellular dopamine concentration in freely moving rats by fast-scan cyclic voltammetry. Methods Mol Med 79:443-464.

Richardson NR, Gratton A (1996) Behavior-relevant changes in nucleus accumbens dopamine transmission elicited by food reinforcement: an electrochemical study in rat. J Neurosci 16:8160-8169.

Robinson DL, Heien MLAV, Wightman RM (2002) Frequency of dopamine transients increases in the dorsal and ventral striatum of male rats during introduction of conspecifics. J Neurosci 22:10477-10486.

Salamone JD (1996) The behavioral neurochemistry of motivation: methodological and conceptual issues in studies of the dynamic activity of nucleus accumbens dopamine. J Neurosci Methods 64:137-149.

Salamone JD, Correa M (2002) Motivational views of reinforcement: implications for understanding the behavioral functions of nucleus accumbens dopamine. Behav Brain Res 137:3-25.
Salamone JD, Arizzi MN, Sandoval MD, Cervone KM, Aberman JE (2002) Dopamine antagonists alter response allocation but do not suppress appetite for food in rats: contrast between the effects of SKF 83566, raclopride, and fenfluramine on a concurrent choice task. Psychopharmacology (Berlin) 160:371-380.

Salamone JD, Correa M, Mingote S, Weber SM (2003) Nucleus accumbens dopamine and the regulation of effort in food-seeking behavior: implications for studies of natural motivation, psychiatry, and drug abuse. J Pharmacol Exp Ther 305:1-8.

Schultz W (1998) Predictive reward signal of dopamine neurons. J Neurophysiol 80:1-27.

Schultz W, Apicella P, Ljungberg T (1993) Responses of monkey dopamine neurons to reward and conditioned stimuli during successive steps of learning a delayed response task. J Neurosci 13:900-913.

Smith-Roe SL, Kelley AE (2000) Coincident activation of NMDA and dopamine D1 receptors within the nucleus accumbens core is required for appetitive instrumental learning. J Neurosci 20:7737-7742.

Stratford TR, Kelley AE (1999) Evidence of a functional relationship between the nucleus accumbens shell and lateral hypothalamus subserving the control of feeding behavior. J Neurosci 19:11040-11048.

Venton BJ, Michael DJ, Wightman RM (2003) Correlation of local changes in extracellular oxygen and $\mathrm{pH}$ that accompany dopaminergic terminal activity in the rat caudate-putamen. J Neurochem 84:373-381.

Waelti P, Dickinson A, Schultz W (2001) Dopamine responses comply with basic assumptions of formal learning theory. Nature 412:43-48.

Wilson C, Nomikos GG, Collu M, Fibiger HC (1995) Dopaminergic correlates of motivated behavior: importance of drive. J Neurosci 15:5169-5178

Wise RA, Spindler J, deWit H, Gerberg GJ (1978) Neuroleptic-induced “anhedonia" in rats: pimozide blocks reward quality of food. Science 201:262-264.

Wyvell CL, Berridge KC (2000) Intra-accumbens amphetamine increases the conditioned incentive salience of sucrose reward: enhancement of reward "wanting" without enhanced "liking" or response reinforcement. J Neurosci 20:8122-8130.

Yavich L, MacDonald E (2000) Dopamine release from pharmacologically distinct storage pools in rat striatum following stimulation at frequency of neuronal bursting. Brain Res 870:73-79. 